\title{
The world through their eyes: The perceptions and lived experience among Capetonian young adults with hereditary visual impairment
}

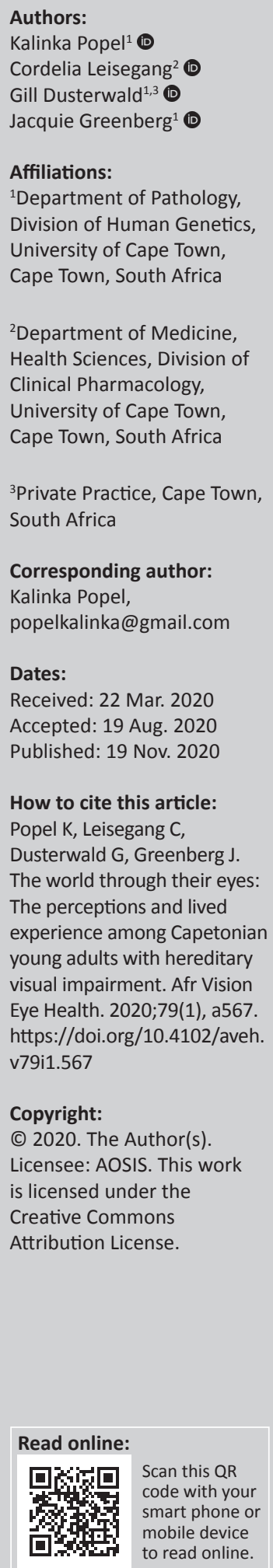

Background: More than one-third of genetic conditions involve the eye. Young adults with visual impairments experience psychosocial implications throughout their lives. The transition from adolescence to adulthood is a critical period during which young adults with hereditary visual impairment make choices regarding relationships, procreation and independence, setting the foundation for their future.

Aim: This study explored the perceptions and lived experiences of visually impaired young adults.

Setting: The study was conducted at three institutions for the blind in Cape Town.

Methods: Using purposive sampling, in-depth qualitative interviews were conducted using a phenomenological approach with 15 young adults aged 18-23 years with genetic-based visual conditions. The data were analysed using thematic analysis.

Results: Eleven participants had minimal knowledge and understanding of their condition, which also created uncertainty about their future health. Making decisions about procreation was the main implication of having a genetic-based visual impairment. Mixed feelings were reported regarding bearing offspring. Social alienation was experienced in some instances; many participants felt misunderstood by society, and intimate relationships were thought to be harder to obtain. Challenges with mobility, such as the inability to drive and the need to use public transportation, significantly impacted them, and various psychosocial aspects influenced how they perceived their lives and future.

Conclusion: This research indicates that improved patient-centred healthcare services and support systems would be beneficial in aiding visually impaired individuals successfully transition into adulthood. The findings of this study serve as a useful guide for those who need to provide healthcare services, genetic services and support for visually impaired young adults.

Keywords: visual impairment; young adults; genetic; qualitative; inherited.

\section{Introduction}

Worldwide, roughly 188 million individuals have mild visual impairment, 217 million have moderate-to-severe visual impairment and 36 million are blind. ${ }^{1}$ In 2001, approximately 2.3 million (5\%) of the 44819778 inhabitants of South Africa had a disability. Visual impairment formed the primary disability group, consisting of 600000 (1.3\%) individuals. ${ }^{2}$ Mathebula notes that more than one-third of genetic conditions involve the eye. ${ }^{3}$ Young adults with visual impairments may experience various psychosocial challenges. ${ }^{4}$ The successful transition from adolescence to adulthood is crucial for visually impaired young adults and society in general, as disability may cause psychological distress and impact the social, psychological and physical potential of these individuals in later life..$^{5}$ This transition encompasses friendships, marriage, parenthood and the capacity to make and execute decisions independently. ${ }^{6}$

Minimal knowledge and understanding of the medical and genetic aspects of their inherited visual impairment has been demonstrated in young adults, regarding their condition, ${ }^{7}$ which often results in misinterpretations about genetic conditions. ${ }^{8,9}$ Furthermore, confusion occurs among some individuals about the risks of having affected children. ${ }^{8}$ In situations where parents 
have had a child with a genetic disorder, it has been found that $34.5 \%$ desired more children, whilst $65.5 \%$ rejected this notion, indicating that procreation decision-making and its consequences lead to ambivalence about procreation. ${ }^{10}$ Research by Petersen ${ }^{11}$ noted that decisions stem from thinking of the well-being of a child versus the desire to have children. The importance of ensuring that young adults have appropriate knowledge and understanding of their genetic condition and risks could equip them to communicate and interact efficiently with family, friends, the community and healthcare professionals throughout life. ${ }^{12}$ This is assisted by genetic counselling, which fills information gaps, helps the individual to reduce psychological anguish and adjust to the disorder more effectively and in this way ensures a smoother transition to adulthood. ${ }^{13}$

Isolation and smaller social networks are not uncommon among the visually impaired because of the discomfort and uncertainty sighted individuals face about communicating with disabled individuals, which leads to exclusion in social activities. ${ }^{14,15}$ Communication breakdown resulting from a reduced capacity to identify visual cues occurs frequently in conversations. Consequently, individuals become demotivated and withdraw from social interactions. ${ }^{16}$ When visually impaired young adults become more self-conscious of their appearance, it becomes increasingly challenging to approach potential suitors. ${ }^{17}$ Furthermore, misconceptions about disabled people and the lack of visual acuity itself are factors contributing to the hurdles visually impaired individuals face in having intimate relationships. ${ }^{18}$ In addition, visually impaired individuals experience challenges with mobility on multiple levels. Independence is often partially represented by obtaining a driver's license and driving; therefore, depending on others for transportation can be frustrating. Employment, social engagement and routine activities are often hindered by limitations related to driving. ${ }^{19}$ Many people rely on public transportation; however, the transport does not always stop at the designated area, and often announcements are not made throughout the commute or stopovers, which results in confusion about which transport to take and when to disembark because of the inability to read bus and road signs. ${ }^{20,21}$ Moreover, uneven surfaces, steps, crossing streets and crowds make outdoor mobility a challenging and dangerous task..$^{20,22}$

Apart from learning more about genetic visual disorders, we need to gain insight on how young adults perceive their disorder and how it affects their lives to create better patientcentred genetic services and more targeted methods and support systems to assist them with their transition to adulthood. Therefore, the aim of this study was to explore the perceptions, understanding and lived experiences of young adults who have genetic-based visual impairments.

\section{Methods}

\section{Study design}

This study used a qualitative design, which utilised a phenomenological approach.

\section{Population and setting}

Participants were recruited via purposive sampling from organisations for individuals with visual impairments in Cape Town, namely, Athlone School for the Blind, Retina South Africa and the League of Friends of the Blind. Information about the study was provided to potential participants at meetings at the various locations. Male and female young adults aged 18-23 years were recruited, who had visual impairment as either the primary result of genetic factors, such as retinitis pigmentosa, or because of eye abnormalities manifesting in association with genetic conditions such as albinism. The visual capacity of the participants ranged from limited vision to legally blind. Individuals who were unaware of the potential genetic component of their visual condition were excluded from the study, to avoid unconsented disclosure and psychological trauma.

\section{Instrumentation and procedures}

A short sociodemographic questionnaire and a semistructured interview guide were developed. Firstly, a test interview, which was conducted with a colleague and then with a visually impaired individual, was analysed to identify and rectify any shortcomings in the questionnaire. The participants signed a consent form and completed the short questionnaire to obtain sociodemographic information. Braille documents were available for participants who required them. Thereafter, face-to-face in-depth interviews were conducted and recorded by the primary researcher, using the open-ended, semi-structured guides. The question guide can be viewed in Table 1.

\section{Data explicitation}

Interviews were transcribed verbatim. To ensure confidentiality, interviews were anonymised using alphanumeric codes (P1-P15) and fictitious names. Thematic analysis was conducted to identify themes. This was done by repeatedly reading the data to become more familiar with the content, and then codes were generated. Themes and subthemes were created by making clusters of the codes, which repeatedly surfaced whilst reading and mapping the interview scripts. To ensure validity, the second author reviewed the codes to identify discrepancies, which were checked by the other authors and addressed accordingly; then, connections were formed between the data and prior literature findings.

\section{Ethical consideration}

The research was reviewed and approved by the Faculty of Health Sciences Health Research Ethics Committee of the University of Cape Town (HREC REF: 376/2016) on 15 July 2016.

Informed consent was obtained from each participant prior to their interview. Participants could opt not to answer a question or to withdraw from the study at any stage. The data was only accessible by the authors of this article. 
TABLE 1: Question guide.

\begin{tabular}{|c|c|}
\hline Key questions & Probes \\
\hline \multirow{6}{*}{$\begin{array}{l}\text { Tell me more about } \\
\text { what it is like being } \\
\text { a person who has a } \\
\text { genetic visual } \\
\text { impairment/blindness? }\end{array}$} & $\begin{array}{l}\text { In what way do you think that your genetic visual condition } \\
\text { affects your relationships/social interactions with people? }\end{array}$ \\
\hline & Finding a partner and being in a relationship? \\
\hline & How do you think society views you? \\
\hline & $\begin{array}{l}\text { What influence has having this genetic visual impairment } \\
\text { had on your education? How about in the future? }\end{array}$ \\
\hline & $\begin{array}{l}\text { Living with genetic visual impairment, what will it mean for } \\
\text { your future? }\end{array}$ \\
\hline & Adjustments/adaptions? \\
\hline \multirow{8}{*}{$\begin{array}{l}\text { What is your } \\
\text { knowledge and } \\
\text { understanding/ } \\
\text { interpretation of } \\
\text { your genetic visual } \\
\text { condition? }\end{array}$} & How do you think you got the condition? \\
\hline & What are the signs/symptoms? \\
\hline & $\begin{array}{l}\text { Do you think other people understand your condition and } \\
\text { situation? Why do you say that? }\end{array}$ \\
\hline & $\begin{array}{l}\text { Have you had any difficulty understanding what your } \\
\text { condition is? }\end{array}$ \\
\hline & $\begin{array}{l}\text { Will it be helpful to you if someone would explain your } \\
\text { condition and the genetic factors regarding your condition to } \\
\text { you? Why? }\end{array}$ \\
\hline & What do you think will happen to your vision in the future? \\
\hline & $\begin{array}{l}\text { How do you think having genetic visual impairment might } \\
\text { impact your health in other ways? For, example, mentally, } \\
\text { physically }\end{array}$ \\
\hline & $\begin{array}{l}\text { How do you think the fact that your disorder is genetic } \\
\text { influences your life? }\end{array}$ \\
\hline \multirow{2}{*}{$\begin{array}{l}\text { Can you describe how } \\
\text { genetic visual } \\
\text { impairment/blindness } \\
\text { has affected your } \\
\text { decision-making around } \\
\text { having children? }\end{array}$} & $\begin{array}{l}\text { What would it be like to have a child/children with the } \\
\text { same condition as you? }\end{array}$ \\
\hline & $\begin{array}{l}\text { What, if anything would you do differently in raising a child } \\
\text { with visual impairment, compared to how you were raised? }\end{array}$ \\
\hline \multirow{2}{*}{$\begin{array}{l}\text { Tell me about your } \\
\text { experience of the } \\
\text { obstacles you have } \\
\text { faced over the last } \\
\text { couple of years? }\end{array}$} & $\begin{array}{l}\text { It sounds like it was difficult getting around - could you } \\
\text { explain a bit more? }\end{array}$ \\
\hline & $\begin{array}{l}\text { What types of things have you found easy and difficult } \\
\text { having genetic visual impairment? }\end{array}$ \\
\hline
\end{tabular}

\section{Results}

\section{Demographic information}

The sociodemographic information has been summarised in Table 2. Fifteen $(N=15)$ young adults participated in the study. Thirteen were partially sighted, ranging from moderate-to-severe visual impairment, whilst two were blind, having no visual capacity. Three had albinism, two had retinal degenerative disorders, two had congenital cataracts, two had congenital glaucoma, two had myopia, one had Peter's anomaly, one had coloboma, one had uveitis and family history of visual impairments, and one had an uncertain diagnosis but a family history of visual impairments and was included on that basis.

The perceptions about the implications of having a hereditary visual impairment became apparent in several themes, namely, understanding of the condition, procreation, social interaction and mobility.

\section{Megatheme 1: Understanding of the condition Lack of knowledge about the condition}

Unclarity or confusion occurred among 11 of the participants regarding what exactly their condition is. This not only created internal turmoil but influenced the way they explained it to others:

'I don't know the genetic disease even. I am not aware of what caused it, what caused me to be blind or anything. I just know
TABLE 2: Participant sociodemographic information

\begin{tabular}{|c|c|c|}
\hline Demographic & $N$ & $\%$ \\
\hline \multicolumn{3}{|l|}{ Gender $(N=15)$} \\
\hline Male & 10 & 66.7 \\
\hline Female & 5 & 33.3 \\
\hline \multicolumn{3}{|l|}{ Age $(N=15)$} \\
\hline 18 & 4 & 26.7 \\
\hline 19 & 7 & 46.6 \\
\hline 20 & 3 & 20.0 \\
\hline 21 & 0 & 0.0 \\
\hline 22 & 1 & 6.7 \\
\hline Mean age & 19.1 & \\
\hline \multicolumn{3}{|l|}{ Current education level $(N=15)$} \\
\hline Grade 10 & 2 & 13.3 \\
\hline Grade 12 & 11 & 73.3 \\
\hline University & 1 & 6.7 \\
\hline NA & 1 & 6.7 \\
\hline \multicolumn{3}{|l|}{ Employment $(N=15)$} \\
\hline Employed & 0 & 0.0 \\
\hline Unemployed & 1 & 6.7 \\
\hline Student - secondary education & 14 & 93.3 \\
\hline \multicolumn{3}{|l|}{ Relationship status $(N=15)$} \\
\hline Single & 13 & 93.3 \\
\hline Informal relationship & 2 & 6.7 \\
\hline \multicolumn{3}{|l|}{ Number of children $(N=15)$} \\
\hline None & 14 & 93.3 \\
\hline One & 1 & 6.7 \\
\hline \multicolumn{3}{|l|}{ Vision status ( $N=15)$} \\
\hline Partially sighted & 13 & 87.0 \\
\hline Blind & 2 & 13.0 \\
\hline \multicolumn{3}{|l|}{ Genetic condition $(N=15)$} \\
\hline Albinism & 3 & 20.0 \\
\hline Retinal degenerative disorder & 2 & 13.0 \\
\hline Congenital cataracts & 2 & 13.0 \\
\hline Congenital glaucoma & 2 & 13.0 \\
\hline Myopia & 2 & 13.0 \\
\hline Coloboma & 1 & 6.7 \\
\hline Peter's anomaly & 1 & 6.7 \\
\hline Uveitis & 1 & 6.7 \\
\hline Uncertain - family history of VI & 1 & 6.7 \\
\hline
\end{tabular}

NA, not applicable; VI, visual impairment.

that I am blind, that's it. So, I don't have any information about it. Even when people ask me why I am blind, then I just say I was born blind, because I don't know the condition of me being blind; I don't know what happened. I was still young.' (Participant 4, 18 years, bilateral uveitis and family history)

\section{What the future holds}

Without concrete knowledge of the condition, they had to guess what the long-term implications would be and how it would impact their lives. Some foresaw visual loss that would lead to significant life changes and challenges:

'My eyes will become weaker, and I think that will be a huge change in my life. I would have to adapt to being blind and all that. I will have to accept that I'm blind, and I will have to go to places to relearn alphabets.' (Participant 2, 19 years, Peters anomaly)

\section{The way in which it is inherited}

The lack of understanding about what caused their visual impairment was further confirmed when 11 of the participants 
were unsure of the way in which they could potentially have acquired their condition. Some had a partial idea, whereas others were very confused:

'I don't understand how it is inherited because I double checked, and the Internet, they said it may be inherited. Which is in my family, somebody that had it; it's not my father, but it's his father - now I think maybe it skipped one generation or two. So, I think obviously now it will skip this generation; I don't know. I don't get it.' (Participant 3, 18 years, congenital glaucoma)

\section{Being misunderstood by others}

Most participants felt misunderstood. The longing for others to understand their condition and what they experienced visually was demonstrated when three participants brought props to the interview in an attempt to explain their visual impairment. Overall, at least 125 statements were made regarding not being understood but desiring understanding:

'Some people, they don't understand, miss.' (Participant 10, 19 years, congenital cataracts)

'Just for them to understand me and fully support me ... it's just the basic thing is to have a good understanding.' (Participant 14, 19 years, bilateral coloboma)

\section{Megatheme 2: Procreation What are the risks involved?}

An internal struggle exists, to determine if they should procreate, because of the uncertainty about the health of their future children. In some instances, their interpretation of the risk of having children with a visual impairment was the driving force of their thought process. This is illustrated by Participant 1, who had the most knowledge about genetic visual conditions:

'I was also told that ... by my doctor, that since it is X-linked and my mother is a carrier, if I have daughters, they would have $80 \%$ of contracting that condition.' (Participant 1, 22 years, retinal degenerative disorder)

\section{Not willing to take the chance}

For some, such as Participant 10, the risk was too big, and they were not willing to take the chance:

'I don't want no kids. Because I am scared, they will be like their dad, you see. I believe that it is going to be unfair to me to give my kids this.' (Participant 10, 19 years, congenital cataracts)

\section{If I can do it, they can do it too}

Over half of the participants, including Participant 11 felt that if they could live with the condition, their children could, too, and as they wanted to be accepted, they would accept their children too:

'I will just accept it the way it is. Because I can't take it back and say, “hey, go back, I don't want you because you are visually impaired like me". I won't throw him away, because I know the situation; I come from that situation.' (Participant 11, 20 years, retinal degenerative disorder)

\section{Megatheme 3: Social interaction I feel left out}

Having a visual impairment has affected their integration into society. Isolation from society, friends and family is often the case among these young adults:

'They see things that I can't see, and they laugh at things that I maybe can't see what they laughing at, so I feel out sometimes, and I just go sit at home.' (Participant 10, 19 years, congenital cataracts)

\section{I do not want them to know that I cannot see}

Some feel self-conscious about their condition and try to hide it from others. For example, Participant 10 hampers his vision by not wearing glasses around sighted individuals:

'I have to wear glasses every day, and that's the thing I can't do. I feel embarrassed. Because I am used to go out to normal people, and many of my friends don't know I have this disability. I keep on hiding it away. I don't want them to see that I am like this.' (Participant 10, 19 years, congenital cataracts)

\section{Some people do not treat me right}

Unempathetic or judgemental responses and treatment from society often alienated these visually disabled individuals:

'We always get people that will, like, try to distance themselves from you and will always try to take advantage from you.' (Participant 11, 20 years, retinal degenerative disorder)

Their psychological well-being was negatively impacted by the bullying that some of them endured. As a result, one participant was even admitted to a mental health facility for a period of time:

'I had depression before; it was basically because of my eyes, and like I have been bullied at the communities.' (Participant 12, 19 years, Glaucoma)

\section{The challenges of finding a partner}

Various feelings surfaced regarding intimate relationships. Two participants felt that a man would fear to marry them because of the risk to their offspring, which is explained by Participant 2. For nine participants, such as Participant 12, their disability in itself was believed to be a barrier in finding a partner, more so than the genetic aspect:

'Some guys may be afraid to be with me because they are afraid that their child will end up the same and stuff.' (Participant 2, 19 years, Peters anomaly)

'I think it is going to be hard because most girls don't want to take guys that has a disability or something like that.' (Participant 12, 19 years, Glaucoma)

A few did not want partners who were visually impaired out of concern of increasing the risk to their offspring:

'I don't want a person who also has a genetic disease because that will increase the chances of me also getting offspring that will also have this genetic condition.' (Participant 4, 18 years, bilateral uveitis) 


\section{Megatheme 4: Mobility Driving independently is not an option}

All the young adults had experienced challenges with mobility in one way or another. All the partially sighted individuals had a strong desire to drive independently. However, many noted that they were unable to do so and depending on others was not ideal:

'I can't drive a car. I have to ask people to drive me certain places, and some people will say no, when it's urgent or something like that.' (Participant 7, 18 years, Albinism)

Surrendering the dream of driving led to unpleasant emotions. The sadness and disappointment were visibly displayed by those who spoke about not being able to drive:

'It's painful because as I mentioned that you can't see, and there will be actually obstacles that are in our way because of the visual impairment. For instance, driving. Like we have to pass a certain test, so what if I did not pass the test, then my dreams of having my own car and driving it will be smashed.' (Participant 9, 18 years, Albinism)

\section{Public transportation is a challenge}

Engaging with the public transportation system is challenging because seeing the information displayed on busses, trains and road signs is difficult, and this often leads to the young adults missing their transport:

'The bus, when they come, you have to look there, right at the front is the name - I can't see that, so the only time I see is when it is nearby, then it's gone, then I have to wait again for another bus.' (Participant 2, 19 years, Peters anomaly)

\section{The public do not make the transportation challenges easier}

A few participants also mentioned the psychological impact of the reactions they got from onlookers. Participant 11 explained that instead of helping, people first questioned and commented on the situation, which discouraged them from asking for help:

'Most people, if you ask them, they are going to ask, "can't you see the writings there?" If you are telling them, like, "no, I can't see, my eyes are a problem". "Oh, wow, you are so young to have a problem with your eyes", and all that stuff. Then it's, like, tough for those people that are shy to ask.' (Participant 11, 20 years, retinal degenerative disorder)

\section{Moving around outside can be dangerous}

Navigating on foot is dangerous, and although one participant mentioned that he had a lot of assistance from community members when he needed to walk somewhere, a few mentioned that they felt unsafe when crossing roads or in dangerous areas. This is demonstrated by the frightening experiences of Participant 7:

'Going over the road, I should be injured right now because of the amount of times I got knocked over. I have been hit by bakkies and other vehicles a bunch of times.' (Participant 7, 18 years, Albinism)

\section{Discussion}

A significant factor that arose in this study was that there was insufficient knowledge and understanding about the condition itself and the reproductive implications. People generally seek clarity on the cause, diagnosis, visual impact, progression, management and recurrence risks of their condition. ${ }^{23}$ Lack of knowledge had created uncertainty, confusion and anxiety for the young adults in this study. This confusion can create an obstacle in identity development, which according to Erikson ${ }^{24}$ is a key developmental stage, and can hinder a smooth transition to adulthood. Pichini et al. ${ }^{25}$ documented a group of young adults who experienced the same uncertainty about their condition. They reported genetic counselling as a beneficial process for coping. The young people in this study must transition to adult healthcare and will benefit from understanding their condition better so that they can claim responsibility for their management and lives. ${ }^{26}$ Furthermore, individuals in the current study, similar to the study by Clarke et al., ${ }^{23}$ were concerned about a decrease in vision and the significant impact it would have on their lives. Many participants in the study illustrated the importance of genetic counselling, noting that it would aid them in attaining more knowledge and clarity, which illustrates its importance.

Deaf individuals have a deaf culture; in contrast, visually impaired individuals do not have a specific blind culture with which they can identify. ${ }^{27}$ This created a sense of displacement, a yearning to be understood and a desire to integrate into the sighted world in most of the individuals in this study. As disabled individuals, including those in this study, often feel misunderstood by society, explaining and interacting with others is often seen as a pointless activity. ${ }^{9,28}$

The participants were unsure about the risks to future offspring, and they displayed mixed feelings about reproducing. Individuals who are affected with or have offspring with a genetic condition perceive reproduction as an unpredictable and risky affair..$^{10}$ For some individuals in this study, the risk factor might have been amplified by inaccurate or limited knowledge about the inheritance risks of the condition. Uninformed decisions regarding reproduction could result in some of the young adults not bearing offspring, despite being at low risk of having an affected child or vice versa. The medical and psychosocial experiences of individuals or their families have been said to also play a role in the construction of risk perception. ${ }^{29}$ In the current study, it was found that the individuals who felt more knowledgeable and in control of their situation had formulated a risk perception that was less threatening, whilst those who were struggling most to cope with their condition had developed a risk perception prohibiting them from procreating.

The social interactions of the young adults had been significantly impacted because of their visual impairment. Being socially isolated and excluded, having few friends, limited social networks and limited capacity to participate in social activities is common among visually impaired 
individuals. ${ }^{9,30,31}$ Visually impaired young adults become more socially isolated as they become more self-conscious about their physical appearance and visual capacity, which hinders them from engaging in social interactions. ${ }^{16}$ This was illustrated by some young adults in the study who withdrew from social situations or had undertaken extreme measures to conceal their impairment. Some young adults in this current study had been subjected to bullying, which, according to Hodge and Eccles, ${ }^{16}$ is a problem highlighted in many studies on visually impaired individuals. To avoid the emotional scars, they avoided social interactions to minimise their vulnerability.

Some anxiety exists about how a potential partner would react towards their condition. Some females in the study, in accordance with Gill, ${ }^{32}$ noted that a non-disabled partner might re-evaluate a relationship with a disabled individual because of the potential risks to offspring, but more of the participants themselves did not want a visually impaired partner for this reason. This could create a hurdle because, according to Gaigher et al. ${ }^{7}$ and Gill, ${ }^{32}$ the chance of finding a sighted partner is decreased because of prejudice towards disabled individuals and the fact that sighted individuals are sometimes frowned upon when having a disabled partner.

The inability to drive limited these individual's freedom and resulted in frustration and despair.,21 Disappointment and heartbreak were displayed by the young adults for not being able to reach their dream of driving independently. In some countries, visually impaired individuals have requested auditory traffic lights because of the challenges faced when attempting to cross the street. ${ }^{21}$ This illustrates the anxiety that the visually impaired young adults also felt. For those with limited vision, like the young adults in this study, the feeling of insecurity is amplified, and many of them are wary of being mobile outdoors. Safety concerns are amplified by visually disabled individuals, including the young adults in this study, when using public transportation. ${ }^{33}$

Mobility courses are often used to acquire efficient mobility; however, physical and mental challenges can still be faced, regardless of learning orientation and mobility skills. ${ }^{22,34}$ This study cohort did not mention orientation and mobility training, although the researcher was aware that this service was provided at the institutions. It is unclear if they did not mention this information because it has not resolved all of their mobility challenges, because they did not think of it at the time of the interview or for other reasons.

\section{Practice implications}

Various connections should be made between support systems, such as from school to organisations for the blind and child healthcare to adult healthcare, during transition into adulthood. Furthermore, this research indicates that better systems should be implemented for appropriate and efficient referrals of individuals with hereditary visual conditions to genetic services to receive genetic counselling, so that they can understand and adapt their condition better.
To provide improved service delivery that meets the specialised needs of the patient, healthcare professionals must be aware of the perceptions of young adults with hereditary visual impairment. More effective public transportation systems or assistive devices need to be designed, such as audio announcements of the bus or other transport and the route. These aspects would be beneficial to all visually impaired individuals, regardless of whether their vision loss is of a genetic nature or not.

\section{Study limitations}

Most participants were recruited from the Athlone School for the Blind, which could create a skewed study sample. Thus, the data might not accurately represent other visually impaired young adults' experiences. Because of the examination period and the academic year reaching closure, the young adults would return to their home towns and would not be able to participate; therefore, the interviews were conducted in batches over a compact period, prohibiting the researcher from thoroughly evaluating and reflecting on the interviewing process after each interview. Opportunities may have been missed to probe further into aspects that were identified later. Most of the participants, who are still in their familiar and protected environment of a secondary schooling system, might experience life differently from those individuals who have already left school and are experiencing situations that average young adult faces; therefore, aspects such as procreation might have been viewed differently if they were older and no longer at school.

\section{Research recommendations}

It would be useful to explore the perceptions of visually impaired young adults who have had genetic counselling to determine the impact of the genetic counselling process on their perceptions. Investigations can be done to determine the similarities and differences in the worldviews of visually impaired individuals who have been affiliated with organisations for the blind versus those who have not. As the young adults feel misunderstood by society, it would be beneficial to research society's perception of visually impaired individuals.

\section{Conclusion}

This research indicates that minimal knowledge and understanding of visual conditions existed among the cohort. The data suggest that knowledge deficits among individuals with genetic disabilities could be perceived to hinder individual identity development and reproduction choices. The integration of genetic counselling services within existing social benefit structures could alleviate the general feeling of uncertainty about the future experienced by young adults with visual impairments. Furthermore, relationships with friends, family and the general society are negatively impacted as a result of the visual impairment, and for some individuals intimate relationships may be difficult to acquire. Mobility tends to 
be a continuous hurdle, regardless of being a pedestrian or using public transportation, which suggests the need for the visually impaired community to take a more active role in urban planning. These findings are a useful guide for those who need to provide healthcare and social services, genetic services and support for visually impaired young adults.

\section{Acknowledgements}

The authors are grateful to the participants who gave their time and shared their experiences. They thank the institutions for the blind, namely, Retina South Africa, Athlone School for the Blind and the League of Friends of the Blind, for granting permission for the participants to be recruited from their members and pupils and for the interviews to be conducted on their premises in most instances.

\section{Competing interests}

The authors declare that they have no financial or personal relationships that may have inappropriately influenced them in writing this article.

\section{Authors' contributions}

K.P. designed the study, wrote the protocol, collected the data, performed the data analysis and wrote the manuscript. J.G., C.L. and G.D. reviewed the protocol and manuscript. J.G. was the main supervisor, and C.L. and G.D. were cosupervisors. All authors approved the final version of the submitted manuscript.

\section{Funding information}

This study was funded by the South African National Research Foundation.

\section{Data availability statement}

Data sharing is not applicable to this article.

\section{Disclaimer}

The views and opinions expressed in this article are those of the authors and do not necessarily reflect the official policy or position of any affiliated agency of the authors.

\section{References}

1. World Health Organization. Blindness and vision impairment [homepage on the Internet]. World Health Organization; 2019 [cited 2019 Jul 15]. Available from: http://www.who.int/mediacentre/factsheets/fs282/en/

2. Sacharowitz HS. Visual impairment in South Africa: Achievements and challenges S Afr Optom. 2005;64(4):139-149. https://doi.org/10.4102/aveh.v64i4.239

3. Mathebula SD. A review of ocular genetics and inherited eye diseases. S Afr Optom. 2012;71(4):178-189. https://doi.org/10.4102/aveh.v71i4.81

4. Gilbert C, Foster A. Childhood blindness in the context of VISION 2020 - The right to sight. Bull World Health Organ. 2001;79(3):227-232.

5. Elsman EBM, Van Rens GHMB, Van Nispen RMA. Quality of life and participation of young adults with a visual impairment aged 18-25 years: Comparison with population norms. Acta Ophthalmol. 2019;97(2):165-172. https://doi.org/10.1111/ aos.13903
6. Clark A, Hirst M. Disability in adulthood: Ten-year follow-up of young people with disabilities. Disabil Handicap Soc. 2007;4(3):271-283. https://doi.org/10.1080/ 02674648966780291

7. Gaigher RJ, Lund PM, Makuya E. A sociological study of children with albinism at a special school in the Limpopo province. Curationis. 2002;25(4):4-11. https://doi. org/10.4102/curationis.v25i4.793

8. Smith M, Aguirre TP. Reproductive attitudes and behaviors in people with sickle cel disease or sickle cell trait: A qualitative interpretive meta-synthesis. Soc Work Health Care. 2012;51(9):757-779. https://doi.org/10.1080/00981389.2012.693580

9. Popel K, Leisegang C, Dusterwald G, Greenberg J. Young adults' perceptions of the implications of their hereditary visual impairment: A Cape Town based study. Cape Town: University of Cape Town; 2017.

10. Kelly SE. Choosing not to choose: Reproductive responses of parents of children with genetic conditions or impairments. Sociol Health IIIn. 2009;31(1):81-97. https://doi.org/10.1111/j.1467-9566.2008.01110.x

11. Petersen $A$. The best experts: The narratives of those who have a genetic condition. Soc Sci Med. 2006;63(1):32-42. https://doi.org/10.1016/j.socscimed.2005.11.068

12. Guerette AR, Lewis $S$, Mattingly C. Students with low vision describe their visual impairments and visual functioning. J Vis Impair Blind. 2011;105(5):287-298. https://doi.org/10.1177/0145482×1110500506

13. Szybowska M, Hewson S, Antle, BJ, Babul-Hirji R. Assessing the informational needs of adolescents with a genetic condition: What do they want to know? J Genet Counsel. 2007;16(2):201-210. https://doi.org/10.1007/s10897-006-9060-5

14. Hersen M, Van Hasselt VB, Segal DL. Social adaption in older visually impaired adults: Some comments. Int J Rehabil Health. 1995;1(1):49-60. https://doi. org/10.1007/bf02214961

15. Jessup GM, Bundy AC, Hancock N, Broom A. Being noticed for the way you are Social inclusion and high school students with vision impairment. Br J Vis Impair. 2017;36(1):90-103. https://doi.org/10.1177/0264619616686396

16. Hodge S, Eccles F. Loneliness, social isolation and sight loss. Lancaster: Thomas Pocklington Trust, Lancaster University; 2013.

17. Pinquart $M$, Pfeiffer J. What is essential is invisible to the eye: Intimate relationships of adolescents with visual impairment. Sex Disabil. 2012;30:139-147. https://doi. org/10.1007/s11195-011-9248-y

18. Fichten CS, Goodrick C, Amsel R, Wicks McKenzie S. Reactions towards dating peers with visual impairments. Rehabil Psychol. 1991;36(3):163-178. https://doi. org/10.1037/0090-5550.36.3.163

19. Owsley C, McGwin G. Vision impairment and driving. Surv Opthalmol. 1999;46(3):535-550. https://doi.org/10.1016/s0039-6257(99)00035-1

20. Arora A, Shetty A. Common problems faced by visually impaired people. Int J Sc Res. 2014;3(10):2002-2005.

21. Golledge RG, Costanzo CM, Marston J. Public transit use by non-driving disabled persons: The case of the blind and vision impaired. PATH Conference; 1995 Oct 13; University of California, Santa Barbara, California.

22. Jeffries JM, Gilroy R, Townshend T. Challenging the visual: Learning from the mobility narratives of visually impaired persons. J Urban Des. 2018;25(2):254-257. https://doi.org/10.1080/13574809.2018.1494503

23. Clarke E, Combs R, Black G, Hall G. Patient expectations and attitudes towards specialized genetic eye services. J Genet Counsel. 2015;24(2):349-357. https:// doi.org/10.1007/s10897-014-9775-7

24. Erikson EH. Identity and the life cycle: Selected papers. New York, NY: International Universities Press; 1959.

25. Pitchini A, Shuman C, Sappleton K, Kaufman M, Chitayat D, Babul-Hirji R. Experience with genetic counseling: The adolescent perspective. J Genet Counsel. 2016;25(3):583-595. https://doi.org/10.1007/s10897-015-9912-y

26. Robertson AO, Tadić $\mathrm{V}$, Rahi JS. Transition from paediatric to adult ophthalmology services: What matters most to young people with visual impairment. Eye. 2018;32:406-414. https://doi.org/10.1038/eye.2017.203

27. Weisleder P. No such thing as a 'blind culture.' J Child Neurol. 2012;27(6):819-820. https://doi.org/10.1177/0883073812441249

28. Brouwer DM, Sadlo G, Winding K, Hanneman MIG. Limitations in mobility: Experiences of visually impaired older people. Br J Occup Ther. 2008;71(10): 414-421. https://doi.org/10.1177/030802260807101003

29. Klitzman RL. Misunderstandings concerning genetics among patients confronting genetic disease. J Genet Counsel. 2010;19(5):430-446. https://doi.org/10.1007/ s10897-010-9307-z

30. Huurre TM, Komulainen EJ, Aro HM. Relationships with parents and friends, selfesteem and depression among adolescents with visual impairments. Scan J Disabil Res. 2001;3(1):21-37. https://doi.org/10.1080/15017410109510766

31. Chan VF, Singer S, Naidoo KS. Disability-related-distress in primary school learners with vision impairment due to uncorrected refractive error in KwaZulu-Natal Province, South Africa - A qualitative study. PLoS One. 2020;15(3):1-16. https:// doi.org/10.1371/journal.pone.0229108

32. Gill CJ. Dating and relationship issues. Sex Disabil. 1996;14(3):183-190. https:// doi.org/10.1007/bf02590076

33. Kruger T, Landman K. Crime and public transport: Designing a safer journey. 26th South African Transport Conference; 2007 July 09-12; CSIR ICC, Pretoria, South Africa.

34. Soong GP, Lovie-Kitchin JE, Brown B. Does mobility performance of visually impaired adults improve immediately after orientation and mobility training? Optom Vis Sci. 2001;78(9):657-666. https://doi.org/10.1097/00006324-200109000-00011 